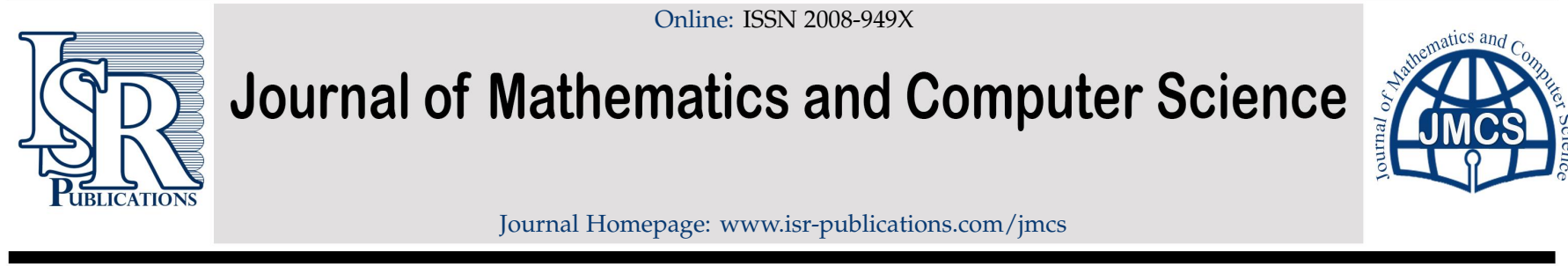

\title{
On the difference between geometric-arithmetic index and atom-bond connectivity index for trees
}

\author{
Wan Nor Nabila Nadia Wan Zuki ${ }^{\mathrm{a}}$, Roslan Hasnia,*, Nor Hafizah Md. Husin ${ }^{\mathrm{a}}$, Zhibin Du $^{\mathrm{b}}$, Abdul Raheem $^{\mathrm{c}}$ \\ ${ }^{a}$ Faculty of Ocean Engineering Technology and Informatics, University Malaysia Terengganu, 21030 Kuala Nerus, Terengganu, \\ Malaysia. \\ ${ }^{b}$ School of Mathematics and Statistics, Zhaoqing University, Zhaoqing, Guangdong, P. R. China. \\ ${ }^{c}$ Department of Higher Education, Govt. Postgraduate College Asghar Mall Rawalpindi, Pakistan.
}

\begin{abstract}
Let $G$ be a simple and connected graph with vertex set $V(G)$ and edge set $E(G)$. The geometric-arithmetic index and atom-bond connectivity index of graph $G$ are defined as $G A(G)=\sum_{u v \in E(G)} \frac{2 \sqrt{d_{u} d_{v}}}{d_{u}+d_{v}}$ and $A B C(G)=\sum u v \in E(G) \sqrt{\frac{d_{u}+d_{v}-2}{d_{u} d_{v}}}$, respectively, where the summation extends over all edges $u v$ of $G$, and $d_{u}$ denotes the degree of vertex $u$ in $G$. Let $(G A-$ $A B C)(G)$ denote the difference between $G A$ and $A B C$ indices of $G$. In this note, we determine $n$-vertex binary trees with first three minimum $G A-A B C$ values. We also present a lower bound for $G A-A B C$ index of molecular trees with fixed number of pendant vertices.
\end{abstract}

Keywords: Atom-bond connectivity index, geometric-arithmetic index, trees.

2020 MSC: 05C35, 05C90.

(C)2021 All rights reserved.

\section{Introduction}

Let $G$ be a simple and connected graph with vertex set $V(G)=\left\{v_{1}, v_{2}, \cdots, v_{n}\right\}$ and edge set $E(G)=$ $\left\{e_{1}, e_{2}, \cdots, e_{n}\right\}$, where $|V(G)|=n$ and $|E(G)|=m$. For $u \in V(G), d_{u}$ denotes the degree of vertex $u$ in G. The minimum degree and maximum degree of $G$ are denoted by $\delta$ and $\Delta$. Let $x, y \in V(G)$, then the distance $d_{G}(x, y)$ between $x$ and $y$ is defined as the length of any shortest path in G connecting $x$ and $y$. For a vertex $v_{i}$ of $V(G)$, its eccentricity $e_{i}$ is the largest distance between $v_{i}$ and any other vertex $v_{k}$ of $G$, i.e., $e_{i}=\max _{v_{j} \in V(G)} d_{G}\left(v_{i}, v_{j}\right)$. For the notations and terminologies not mentioned here, please refer to [19].

Graph theory has provided the chemists with a variety of useful tools, one of which is the topological indices [7]. Molecules and molecular compounds are often modeled by molecular graphs. Topological indices of molecular graphs are one of the oldest and the most widely used descriptors in QSPR/QSAR research [16].

\footnotetext{
*Corresponding author

Email address: hroslan@umt.edu.my (Roslan Hasni)

doi: $10.22436 /$ jmcs.022.01.05
}

Received: 2020-04-16 Revised: 2020-04-28 Accepted: 2020-05-16 
Estrada et al. [10] proposed the atom-bond connectivity index of graph $\mathrm{G}$ which is abbreviated as $\mathrm{ABC}(\mathrm{G})$ and defined as

$$
A B C(G)=\sum_{u v \in E(G)} \sqrt{\frac{d_{\mathfrak{u}}+d_{v}-2}{d_{u} d_{v}}},
$$

where the summation goes over all edges of G. The ABC index is one of many so called topological indices that are extensively used in theoritical chemistry to correlate physico-chemical properties with the molecular structures of chemical compounds. Its appear that the ABC index shows a strong correlation with heat of formation of alkanes [10]. Some topological approaches were also developed based on the $A B C$ index to explain the differences in the energy of linear and branched alkanes [9].

In the study of topological indices in general, it is often of interest to consider the extremal values of a certain index among graphs under various constrains. Along this line, the extremal values of $A B C$ index have been extensively explored, see $[2,8,11,15,20]$ and the references cited therein.

Another topological index of graph G, named the geometric-arithmetic index and abbreviated as $G A(G)$, was introduced by Vukičević and Furtula [17] in 2009. The GA index is defined as follows

$$
G A(G)=\sum_{u v \in E(G)} \frac{2 \sqrt{d_{u} d_{v}}}{d_{\mathfrak{u}}+d_{v}}
$$

where the summation goes over all edges of G. It is noted in that the GA index is well correlated with a variety of physico-chemical properties and the predictive power of GA index is somewhat better than the Randić index. Until now, many mathematical properties of GA index were investigated, see the survey [5], some recent papers $[3,4,14,21]$ and the references cited therein.

In line with the great interest of topological indices, some researchers are also keen on the research of the relationship or comparison between topological indices. Das and Trinajstić [6] compared the GA index and $A B C$ index for chemical trees, molecular trees and simple graphs with some restricted conditions. Raza et al. [13] proved that GA index is greater than ABC index for line graphs of molecular graphs, for general graphs in which the difference between maximum and minimum degree is less than or equal to $(2 \delta-1)^{2}$ and for some families of trees. Zhong and Cui [22] obtained a relation between these two indices for simple connected graphs on $n \geqslant 3$ vertices with minimum degree at least $s$ and maximum degree at most $t$, where $1 \leqslant s \leqslant t \leqslant n-1$ and $t \geqslant 2$. Using this relation, they proved that if $t \leqslant 4 s^{2}-3 s+1$, then the $A B C$ index is always less than the GA index and this bound is best possible for $s \geqslant 2$. Recently, Ke [12] and Wei et al. [18] compared the expected values of the GA index and ABC index for random polyphenyl chains and spiro chains, respectively. However, the comparison between these two indices, in the case of trees and general graphs, remains an open problem.

A tree $T$ is said to be a binary tree (chemical tree, respectively) if the maximum vertex degree in $T$ is at most 3 (4, respectively). Very recently, Ali and Du [1] established some extremal results with respect to the difference between $A B C$ index and Randić index ( $R$ index for short) for binary and chemical trees. Let $(G A-A B C)(G)$ be the difference between $G A$ index and $A B C$ index of $G$. In this paper, we investigate the $n$-vertex binary trees with minimum $G A-A B C$ values. We also obtain a lower bound for $G A-A B C$ index of molecular trees with fixed number of pendant vertices.

\section{Minimum values of $G A-A B C$ index for binary trees}

In this section, we characterize the trees with extremal $G A-A B C$ values among all $n$-vertex binary trees.

Let $x_{i, j}$ be the number of edges in the graph $G$ connecting the vertices of degrees $i$ and $j$. Then the formula for the graph invariant $G A-A B C$ can be rewritten in terms of $x_{i, j}$ as follows

$$
(G A-A B C)(G)=\sum_{\delta \leqslant i \leqslant j \leqslant \Delta} \frac{2 i j-(i+j)(\sqrt{i+j-2})}{(\sqrt{i j})(i+j)} x_{i, j}
$$


where $\delta$ and $\Delta$ are, respectively, the minimum and maximum degrees in G.

Let $n_{i}$ be the number of vertices of degree $i$ in $G$. Then the following system of equations holds for any $n$-vertex binary tree $T$ (see $[1,20])$ :

$$
\begin{gathered}
n_{1}+n_{2}+n_{3}=n, \\
n_{1}+2 n_{2}+3 n_{3}=2(n-1), \\
x_{1,2}+x_{1,3}=n_{1} \\
x_{1,2}+2 x_{2,2}+x_{2,3}=2 n_{2} \\
x_{1,3}+x_{2,3}+2 x_{3,3}=3 n_{3} .
\end{gathered}
$$

We solve the system of (2.2), (2.3), (2.4), (2.5), (2.6) for the unknown $n_{1}, n_{2}, n_{3}, x_{1,3}, x_{3,3}$. The values of $x_{1,3}$ and $x_{3,3}$ are, respectively, given as below:

$$
\begin{aligned}
& x_{1,3}=\frac{1}{4}\left(2 n+4-5 x_{1,2}-2 x_{2,2}-x_{2,3}\right), \\
& x_{3,3}=\frac{1}{4}\left(2 n-8+x_{1,2}-2 x_{2,2}-3 x_{2,3}\right) .
\end{aligned}
$$

By substituting these values of $x_{1,3}$ and $x_{3,3}$ in (2.1), one has

$$
\begin{aligned}
(G A-A B C)(G)= & \frac{3 \sqrt{3}-2 \sqrt{6}+2}{12} n+\frac{3 \sqrt{3}-2 \sqrt{6}-4}{6} \\
& -\frac{15 \sqrt{3}-4 \sqrt{2}-10 \sqrt{6}-2}{24} x_{1,2}-\frac{3 \sqrt{3}-2 \sqrt{6}+6 \sqrt{2}-10}{12} x_{2,2} \\
& -\frac{15 \sqrt{3}+60 \sqrt{2}-58 \sqrt{6}+30}{120} x_{2,3} .
\end{aligned}
$$

Let

$$
\begin{aligned}
\wedge= & \frac{15 \sqrt{3}-4 \sqrt{2}-10 \sqrt{6}-2}{24} x_{1,2}+\frac{3 \sqrt{3}-2 \sqrt{6}+6 \sqrt{2}-10}{12} x_{2,2} \\
& +\frac{15 \sqrt{3}+60 \sqrt{2}-58 \sqrt{6}+30}{120} x_{2,3} .
\end{aligned}
$$

Then (2.8) becomes to

$$
(G A-A B C)(G)=\frac{3 \sqrt{3}-2 \sqrt{6}+2}{12} n+\frac{3 \sqrt{3}-2 \sqrt{6}-4}{6}-\wedge
$$

We note that

$$
\begin{aligned}
\frac{15 \sqrt{3}-4 \sqrt{2}-10 \sqrt{6}-2}{24} & \approx-0.2571245652 \\
\frac{3 \sqrt{3}-2 \sqrt{6}+6 \sqrt{2}-10}{12} & \approx-0.1014621407 \\
\frac{15 \sqrt{3}+60 \sqrt{2}-58 \sqrt{6}+30}{120} & \approx-0.01030691021 .
\end{aligned}
$$

So to minimize $(A B C-R)(G)$, it is sufficient to search binary trees for which $\wedge$ is maximum.

Now we present our main result. 
Theorem 2.1. Consider the set of all $\mathrm{n}$-vertex binary trees.

(1) Suppose that $\mathrm{n}$ is even.

(1.1) For $\mathrm{n} \geqslant 4$, the minimum $\mathrm{GA}-\mathrm{ABC}$ value is

$$
\frac{3 \sqrt{3}-2 \sqrt{6}+2}{12} n+\frac{3 \sqrt{3}-2 \sqrt{6}-4}{6}
$$

which is uniquely obtained by those trees that contain no vertex of degree 2 , that is, $\mathrm{n}_{2}=0$.

(1.2) For $\mathrm{n} \geqslant 10$, the second minimum $\mathrm{GA}-\mathrm{ABC}$ value is

$$
\frac{3 \sqrt{3}-2 \sqrt{6}+2}{12} n+\frac{24 \sqrt{6}-30 \sqrt{2}-25}{15},
$$

which is uniquely obtained by those trees that contain exactly two vertices of degree 2 , that is, $\mathrm{n}_{2}=2$, either of which is adjacent to two vertices of degree 3 , that is, $x_{1,2}=x_{2,2}=0$ and $x_{2,3}=4$.

(1.3) For $\mathrm{n} \geqslant 16$, the third minimum GA - ABC value is

$$
\frac{3 \sqrt{3}-2 \sqrt{6}+2}{12} n+\frac{106 \sqrt{6}-15 \sqrt{3}-120 \sqrt{2}-80}{30},
$$

which is uniquely obtained by those trees that contain exactly four vertices of degree 2 , that is, $\mathrm{n}_{2}=4$, either of which is adjacent to two vertices of degree 3 , that is, $x_{1,2}=x_{2,2}=0$ and $x_{2,3}=8$.

(2) Suppose that $\mathrm{n}$ is odd.

(2.1) For $\mathrm{n} \geqslant 7$, the minimum $\mathrm{GA}-\mathrm{ABC}$ value is

$$
\frac{3 \sqrt{3}-2 \sqrt{6}+2}{12} n+\frac{15 \sqrt{3}+38 \sqrt{6}-60 \sqrt{2}-70}{60},
$$

which is uniquely obtained by those trees that contain exactly one vertex of degree 2 , that is, $\mathrm{n}_{2}=1$, which is adjacent to two vertices of degree 3 , that is, $x_{1,2}=0$ and $x_{2,3}=2$.

(2.2) For $\mathrm{n} \geqslant 13$, the second minimum $\mathrm{GA}-\mathrm{ABC}$ value is

$$
\frac{3 \sqrt{3}-2 \sqrt{6}+2}{12} n+\frac{154 \sqrt{6}-15 \sqrt{3}-180 \sqrt{2}-130}{60},
$$

which is uniquely obtained by those trees that contain exactly three vertices of degree 2 , that is, $\mathrm{n}_{2}=3$, each of which is adjacent to two vertices of degree 3 , that is, $x_{1,2}=x_{2,2}=0$ and $x_{2,3}=6$.

(2.3) For $n \geqslant 19$, the third minimum GA - ABC value is

$$
\frac{3 \sqrt{3}-2 \sqrt{6}+2}{12} n+\frac{270 \sqrt{6}-45 \sqrt{3}-300 \sqrt{2}-190}{60},
$$

which is uniquely obtained by those trees that contain exactly five vertices of degree 2 , that is, $\mathrm{n}_{2}=5$, each of which is adjacent to two vertices of degree 3 , that is, $x_{1,2}=x_{2,2}=0$ and $x_{2,3}=10$.

Proof. If $x_{1,2} \geqslant 1$, then by (2.9),

$$
\wedge \geqslant \frac{15 \sqrt{3}-4 \sqrt{2}-10 \sqrt{6}-2}{24} \approx-0.2571245652 .
$$

If $x_{2,2} \geqslant 1$, then by (2.9),

$$
\begin{aligned}
\Lambda & \geqslant \frac{3 \sqrt{3}-2 \sqrt{6}+6 \sqrt{2}-10}{12}+\left(2 \mathrm{n}_{2}-2\right) \cdot \frac{15 \sqrt{3}+60 \sqrt{2}-58 \sqrt{6}+30}{120} \\
& \approx-0.1014621407-0.02061382043 \mathrm{n}_{2}+0.02061382043
\end{aligned}
$$




$$
\begin{aligned}
& \approx-0.02061382043 n_{2}-0.08084832027 \\
& \geqslant \begin{cases}-0.02061382043 \cdot 2-0.08084832027 \approx-0.1220759611, & \text { if } n_{2} \text { is even, } \\
-0.02061382043 \cdot 3-0.08084832027 \approx-0.1426897816, & \text { if } n_{2} \text { is odd. }\end{cases}
\end{aligned}
$$

Suppose in the following that $x_{1,2}=x_{2,2}=0$. Then $x_{2,3}=2 n_{2}$ from (2.5).

Also from (2.7), we have that $n \geqslant \frac{3 x_{2,3}+8}{2}$. From (2.2) and (2.3), we have

$$
n_{2}+2 n_{3}=n-2,
$$

which implies that $n_{2}$ and $n$ have the same parity.

Case 1. $n$ is even.

In this case, note that $n_{2}$ is also even.

If $n_{2}=0$, then $x_{2,3}=0, n \geqslant 4$ and by (2.9), we have $\wedge=0$.

If $n_{2}=2$, then $x_{2,3}=4, n \geqslant 10$ and by (2.9), we have

$$
\wedge=4 \cdot \frac{15 \sqrt{3}+60 \sqrt{2}-58 \sqrt{6}+30}{120} \approx-0.04122764085 .
$$

If $n_{2}=4$, then $x_{2,3}=8, n \geqslant 16$ and by (2.9), we have

$$
\wedge=8 \cdot \frac{15 \sqrt{3}+60 \sqrt{2}-58 \sqrt{6}+30}{120} \approx-0.0824552817
$$

If $n_{2} \geqslant 6$, then $x_{2,3} \geqslant 12$ and by (2.9), we have

$$
\wedge \geqslant 12 \cdot \frac{15 \sqrt{3}+60 \sqrt{2}-58 \sqrt{6}+30}{120} \approx-0.1236829226 .
$$

The first three maximum $\wedge$ are $0,-0.04122764085,-0.0824552817$ and thus the first three minimum $\mathrm{GA}-\mathrm{ABC}$ values for $\mathrm{n}$ is even follow from (2.10) easily.

Case 2. $n$ is odd.

In this case, note that $n_{2}$ is also odd.

If $n_{2}=1$, then $x_{2,3}=2, n \geqslant 7$ and by (2.9), we have

$$
\wedge=2 \cdot \frac{15 \sqrt{3}+60 \sqrt{2}-58 \sqrt{6}+30}{120} \approx-0.02061382043 .
$$

If $n_{2}=3$, then $x_{2,3}=6, n \geqslant 13$ and by (2.9), we have

$$
\wedge=6 \cdot \frac{15 \sqrt{3}+60 \sqrt{2}-58 \sqrt{6}+30}{120} \approx-0.06184146128 .
$$

If $n_{2}=5$, then $x_{2,3}=10, n \geqslant 19$ and by (2.9), we have

$$
\wedge=10 \cdot \frac{15 \sqrt{3}+60 \sqrt{2}-58 \sqrt{6}+30}{120} \approx-0.1030691021 .
$$

If $n_{2} \geqslant 7$, then $x_{2,3} \geqslant 14$ and by (2.9), we have

$$
\wedge \geqslant 14 \cdot \frac{15 \sqrt{3}+60 \sqrt{2}-58 \sqrt{6}+30}{120} \approx-0.144296743
$$

The first three maximum $\wedge$ are $-0.02061382043,-0.06184146128,-0.1030691021$ and thus the first three minimum $G A-A B C$ values for $n$ is odd follow from (2.10) easily.

The binary trees in Theorem 2.1 with the smallest number of vertices are listed in Figures 1 and 2. 


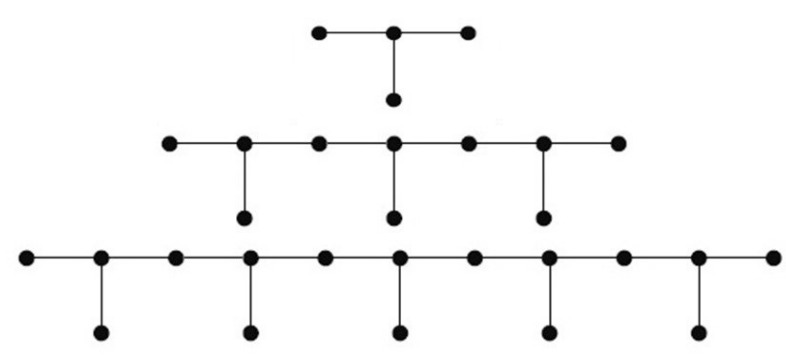

Figure 1: The binary trees in Theorem $2.1(1.1),(1.2)$ and (1.3) with $n=4,10,16$, respectively.

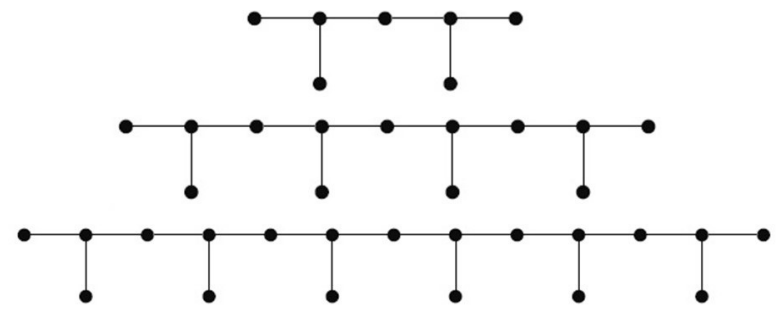

Figure 2: The binary trees in Theorem $2.1(2.1),(2.2)$ and (2.3) with $n=7,13,19$, respectively.

\section{Upper bound for $\mathrm{GA}-\mathrm{ABC}$ index of molecular trees}

For a molecular tree $T$ on $n$ vertices, denote by $n_{i}$ the number of vertices in $T$ with degree $i$ for $i=1,2,3,4$. The following system of equations holds for any $\mathrm{T}$ (see $[1,20])$ :

$$
\begin{gathered}
n_{1}+n_{2}+n_{3}+n_{4}=n \\
n_{1}+2 n_{2}+3 n_{3}+4 n_{4}=2(n-1), \\
x_{1,2}+x_{1,3}+x_{1,4}=n_{1} \\
x_{1,2}+2 x_{2,2}+x_{2,3}+x_{2,4}=2 n_{2} \\
x_{1,3}+x_{2,3}+2 x_{3,3}+x_{3,4}=3 n_{3} \\
x_{1,4}+x_{2,4}+x_{3,4}+2 x_{4,4}=4 n_{4} .
\end{gathered}
$$

Let $\mathcal{T}_{n, n_{1}}$ be the set of molecular trees with satisfy

$$
\begin{gathered}
\mathrm{x}_{1,4}=\mathrm{n}_{1}, \\
\mathrm{x}_{2,2}=\mathrm{n}-2 \mathrm{n}_{1}+3-\frac{1}{3} \mathrm{x}_{2,3}, \\
\mathrm{x}_{2,4}=\mathrm{n}_{1}-4-\frac{2}{3} \mathrm{x}_{2,3}, \\
\mathrm{n}_{2}=\mathrm{n}-\frac{3}{2} \mathrm{n}_{1}+1-\frac{1}{6} \mathrm{x}_{2,3}, \\
\mathrm{n}_{3}=\frac{1}{3} \mathrm{x}_{2,3},
\end{gathered}
$$


and

$$
\mathrm{n}_{4}=\frac{1}{2} \mathrm{n}_{1}-1-\frac{1}{6} \mathrm{x}_{2,3}
$$

Theorem 3.1 ([20]). Let $\mathrm{T}$ be a molecular tree with $\mathrm{n}$ vertices, $\mathrm{n}_{1} \geqslant 5$ of which are pendant vertices. Then

$$
A B C(G) \leqslant \frac{\sqrt{2}}{2} n+\frac{\sqrt{3}-\sqrt{2}}{2} n_{1}-\frac{\sqrt{2}}{2}
$$

with equality holds if and only if $\mathrm{T} \in \mathcal{T}_{\mathrm{n}, \mathrm{n}_{1}}$.

Obviously, from (3.1) we obtain

$$
\begin{aligned}
(G A-A B C)(T)= & \frac{1}{3 \sqrt{2}} x_{1,2}+\frac{6-4 \sqrt{2}}{4 \sqrt{3}} x_{1,3}+\frac{8-5 \sqrt{3}}{10} x_{1,4}+\frac{8-4 \sqrt{2}}{8} x_{2,2} \\
& +\frac{12-5 \sqrt{3}}{5 \sqrt{6}} x_{2,3}+\frac{2}{3 \sqrt{8}} x_{2,4}+\frac{1}{3} x_{3,3}+\frac{24-7 \sqrt{5}}{7 \sqrt{12}} x_{3,4} \\
& +\frac{32-8 \sqrt{6}}{32} x_{4,4} .
\end{aligned}
$$

Now let $\mathcal{T}_{n, n_{1}}^{\prime}$ be the set of molecular trees with satisfy

$$
\begin{gathered}
\mathrm{x}_{1,4}=\mathrm{n}_{1}, \\
\mathrm{x}_{2,2}=\mathrm{n}-2 \mathrm{n}_{1}+3, \\
\mathrm{x}_{2,4}=\mathrm{n}_{1}-4, \\
\mathrm{n}_{2}=\mathrm{n}-\frac{3}{2} \mathrm{n}_{1}+1, \\
\mathrm{n}_{3}=0,
\end{gathered}
$$

and

$$
\mathrm{n}_{4}=\frac{1}{2} \mathrm{n}_{1}-1
$$

Motivated by Theorem 3.1, we prove the following result.

Theorem 3.2. Let $\mathrm{T}$ be a molecular tree with $\mathrm{n}$ vertices, $\mathrm{n}_{1} \geqslant 5$ of which are pendant vertices. Then

$$
(G A-A B C)(T) \geqslant \frac{2-\sqrt{2}}{2} n+\frac{35 \sqrt{2}-15 \sqrt{3}-30}{30} n_{1}+\frac{18-13 \sqrt{2}}{6},
$$

with equality holds if and only if $\mathrm{T} \in \mathcal{T}_{\mathrm{n}, \mathrm{n}_{1}}^{\prime}$.

Proof. Since T is a molecular tree, we have (3.1). Suppose

$$
\begin{aligned}
& f_{1}=x_{1,2}+x_{1,3}+x_{1,4} \\
& f_{2}=x_{1,2}+x_{2,3} \\
& f_{3}=x_{1,3}+x_{2,3}+2 x_{3,3}+x_{3,4} \\
& f_{4}=x_{1,4}+x_{3,4}+2 x_{4,4}
\end{aligned}
$$

that is,

$$
\mathrm{f}_{1}=\mathrm{n}_{1}
$$




$$
\begin{aligned}
& f_{2}=2 n_{2}-2 x_{2,2}-x_{2,4} \\
& f_{3}=3 n_{3}, \\
& f_{4}=4 n_{4}-x_{2,4}
\end{aligned}
$$

we have

$$
\begin{aligned}
\sum_{i=1}^{4} f_{i} & =2(n-1)-2\left(x_{2,2}+x_{2,4}\right), \\
\sum_{i=1}^{4} \frac{1}{i} f_{i} & =n-\left(x_{2,2}+\frac{3}{4} x_{2,4}\right),
\end{aligned}
$$

implying that

$$
\begin{aligned}
& x_{2,2}=\frac{3}{2} \sum_{i=1}^{4} f_{i}-4 \sum_{i=1}^{4} \frac{1}{i} f_{i}+n+3, \\
& x_{2,4}=-2 \sum_{i=1}^{4} f_{i}+4 \sum_{i=1}^{4} \frac{1}{i} f_{i}-4 .
\end{aligned}
$$

Thus we have

$$
\begin{aligned}
& x_{1,4}=n_{1}-x_{1,2}-x_{1,3} \\
& x_{2,2}=n-2 n_{1}+3-x_{1,2}-\frac{1}{3} x_{1,3}-\frac{1}{3} x_{2,3}+\frac{1}{3} x_{3,3}+\frac{2}{3} x_{3,4}+x_{4,4}, \\
& x_{2,4}=n_{1}-4+x_{1,2}+\frac{1}{3} x_{1,3}-\frac{2}{3} x_{2,3}-\frac{4}{3} x_{3,3}-\frac{5}{3} x_{3,4}-2 x_{4,4} .
\end{aligned}
$$

Substituting them back into (3.2), we have

$$
\begin{aligned}
(\mathrm{GA}-\mathrm{ABC})(\mathrm{T})= & \frac{2-\sqrt{2}}{2} \mathrm{n}+\left[\frac{8-5 \sqrt{3}}{10}-\left(\frac{8-4 \sqrt{2}}{4}\right)+\frac{2}{3 \sqrt{8}}\right] \mathrm{n}_{1}+\left(\frac{24-12 \sqrt{2}}{8}-\frac{8}{3 \sqrt{8}}\right) \\
& +\left[\frac{1}{3 \sqrt{2}}-\left(\frac{8-5 \sqrt{3}}{10}\right)-\left(\frac{8-4 \sqrt{2}}{8}\right)+\frac{2}{3 \sqrt{8}}\right] \mathrm{x}_{1,2} \\
& +\left[\frac{6-4 \sqrt{2}}{4 \sqrt{3}}-\left(\frac{8-5 \sqrt{3}}{10}\right)-\left(\frac{8-4 \sqrt{2}}{24}\right)+\frac{2}{9 \sqrt{8}}\right] \mathrm{x}_{1,3} \\
& +\left[\frac{4 \sqrt{2}-8}{24}+\frac{12-5 \sqrt{3}}{5 \sqrt{6}}-\frac{4}{9 \sqrt{8}}\right] \mathrm{x}_{2,3}+\left[\frac{8-4 \sqrt{2}}{24}-\frac{8}{9 \sqrt{8}}+\frac{1}{3}\right] \mathrm{x}_{3,3} \\
& {\left[\frac{16-8 \sqrt{2}}{24}-\frac{10}{9 \sqrt{8}}+\frac{24-7 \sqrt{5}}{7 \sqrt{12}}\right] \mathrm{x}_{3,4}+\left[\frac{8-4 \sqrt{2}}{8}-\frac{4}{3 \sqrt{8}}+\frac{32-8 \sqrt{6}}{32}\right] \mathrm{x}_{4,4} } \\
= & \frac{2-\sqrt{2}}{2} \mathrm{n}+\frac{35 \sqrt{2}-15 \sqrt{3}-30}{30} \mathrm{n}_{1}+\frac{18-13 \sqrt{2}}{6} \\
& +\frac{15 \sqrt{3}+25 \sqrt{2}-54}{30} \mathrm{x}_{1,2}+\frac{45 \sqrt{3}+10 \sqrt{2}-15 \sqrt{6}-51}{45} \mathrm{x}_{1,3} \\
& +\frac{18 \sqrt{6}-20 \sqrt{2}-15}{45} \mathrm{x}_{2,3}+\frac{12-7 \sqrt{2}}{18} x_{3,3} \\
& +\frac{84+72 \sqrt{3}-77 \sqrt{2}-21 \sqrt{15}}{126} \mathrm{x}_{3,4}+\frac{24-10 \sqrt{2}-3 \sqrt{6}}{12} \mathrm{x}_{4,4}
\end{aligned}
$$




$$
\begin{aligned}
= & \frac{2-\sqrt{2}}{2} \mathrm{n}+\frac{35 \sqrt{2}-15 \sqrt{3}-30}{30} n_{1}+\frac{18-13 \sqrt{2}}{6} \\
& +0.244536705 x_{1,2}+0.096490573 x_{1,3}+0.017923202 x_{2,3} \\
& +0.116694725 x_{3,3}+0.146671139 x_{3,4}+0.209116262 x_{4,4}
\end{aligned}
$$

with positive coefficients $x_{1,2}, x_{1,3}, x_{2,3}, x_{3,3}, x_{3,4}$ and $x_{4,4}$. Thus

$$
(G A-A B C)(T) \geqslant \frac{2-\sqrt{2}}{2} n+\frac{35 \sqrt{2}-15 \sqrt{3}-30}{30} n_{1}+\frac{18-13 \sqrt{2}}{6},
$$

with equality holds if and only if the parameters $x_{1,2}, x_{1,3}, x_{2,3}, x_{3,3}, x_{3,4}$ and $x_{4,4}$ are all equal to zero, or equivalently, $x_{1,4}=n_{1}, x_{2,2}=n-2 n_{1}+3, x_{2,4}=n_{1}-4, n_{2}=n-\frac{3}{2} n_{1}+1, n_{3}=0$ and $n_{4}=\frac{1}{2} n_{1}-1$, i.e., $\mathrm{T} \in \mathcal{T}_{\mathrm{n}, \mathrm{n}_{1}}^{\prime}$.

\section{Conclusions}

In this paper, we characterized the first three minimum values of $G A-A B C$ index, where $G A$ and $A B C$ are the geometric-arithmetic index and atom-bond connectivity index, respectively. In particular, we characterized the first, the second and the third minimum n-vertex binary trees with respect to the invariant $G A-A B C$. We also obtained a sharp lower bound for the $G A-A B C$ index of molecular trees with fixed number of pendant vertices.

\section{Acknowledgment}

The authors would like to thank the anonymous referees for their valuable comments and constructive comments that improve the paper. This research is supported by the Research Intensified Grant Scheme (RIGS), Phase 1/2019, Universiti Malaysia Terengganu, Malaysia with Grant Vot. 55192/6.

\section{References}

[1] A. Ali, Z. Du, On the difference between atom-bond connectivity index and Randić index of binary and chemical trees, Int. J. Quantum Chem. 117 (2017), 15 pages. 1, 2, 3

[2] Q. Cui, Q. P. Qian, L. P. Zhong, The maximum atom-bond connectivity index for graphs with edge-connectivity one, Discrete Appl. Math., 220 (2017), 170-173. 1

[3] K. C. Das, On geometric-arithmetic index of graphs, MATCH Commun. Math. Comput. Chem., 64 (2010), 619-630. 1

[4] K. C. Das, I. Gutman, B. Furtula, On the first geometric-arithmetic index of graphs, Discrete Appl. Math., 159 (2011), 2030-2037. 1

[5] K. C. Das, I. Gutman, B. Furtula, Survey on geometric-arithmetic indices of graphs, MATCH Commun. Math. Comput. Chem., 65 (2011), 595-644. 1

[6] K. C. Das, N. Trinajstić, Comparison between the first geometric-arithmetic index and atom-bond connectivity index, Chem. Phsy. Lett., 497 (2010), 149-151. 1

[7] J. Devillers, A. T. Balaban, Topological indices and related descriptors in QSAR and QSPAR, Gordon and Breach, Amsterdam, (1999). 1

[8] D. Dimitrov, On the structural properties of trees with minimal atom-bond connectivity index III: Bounds on $\mathrm{B}_{1}$ - and B -branches, Discrete Appl. Math., 204 (2016), 90-116. 1

[9] E. Estrada, Atom-bond connectivity and energetic of branched alkanes, Chem. Phsy. Lett., 463 (2008), 422-425. 1

[10] E. Estrada, L. Torres, L. Rodriguez, I. Gutman, An atom-bond connectivity index: modelling the enthalpy of formation of alkanes, Indian J. Chem. Sect. A, 37 (1998), 849-855. 1

[11] Y. Gao, Y. Shao, The smallest ABC index of trees with $\mathrm{n}$ pendant vertices, MATCH Commun. Math. Comput. Chem., 76 (2016), 141-158. 1

[12] X. Ke, Atom-bond connectivity and geometric-arithmetic indices in random polyphenyl chain, submitted. 1

[13] Z. Raza, A. A. Bhatti, A. Ali, More comparison between the first geometric-arithmetic index and atom-bond connectivity index, Miskolc Math. Notes, 17 (2016), 561-570. 1

[14] J. M. Rodriguez, J. M. Sigarreta, Spectral properties of geometric-arithmetic index, Appl. Math. Comput., 277 (2016), 142-153. 1

[15] Z. H. Shao, P. Wu, Y. Y. Gao, I. Gutman, X. J. Zhang, On the maximum ABC index of graphs without pendent vertices, Appl. Math. Comput., 315 (2017), 298-312. 1 
[16] R. Todeschini, V. Consonni, Handbook of molecular descriptors, Wiley-VCH, Weinheim, (2000). 1

[17] D. Vukičević, B. Furtula, Topological index based on the ratios of geometrical and arithmetical means of end-vertex degrees of edges, J. Math. Chem., 46 (2009), 1369-1376. 1

[18] S. L. Wei, X. L. Ke, G. L. Hao, Comparing the excepted values of atom-bond connectivity and geometric-arithmetic indices in random spiro chains, J. Inequal. Appl., 2018 (2018), 11 pages. 1

[19] D. B. West, Introduction to Graph Theory, Prentice-Hall, Upper Saddle River, (2001). 1

[20] R. Xing, B. Zhou, F. M. Dong, On the atom-bond connectivity index of connected graphs, Discrete Appl. Math., 159 (2011), 1617-1630. 1, 2, 3, 3.1

[21] Y. Yuan, B. Zhou, N. Trinajstić, On geometric-arithmetic index, J. Math. Chem., 47 (2010), 833-841. 1

[22] L. P. Zhong, Q. Cui, On a relation between the atom-bond connectivity and the first geometric-arithmetic indices, Discrete Appl. Math., 185 (2015), 249-253. 1 